\title{
Upcoming translational challenges for uveal melanoma
}

Nabil Amirouchene-Angelozzi, Marie Schoumacher, Marc-Henri Stern, Nathalie Cassoux, Laurence Desjardins, Sophie

Piperno-Neumann, Olivier Lantz and Sergio Roman-Roman

Correction to: British Journal of Cancer (2015) 113, 1249-1253; doi:10.1038/

bjc.2015.269; published online 27 October 2015

First names and surnames had been inverted. The list of authors has been corrected above.

Upon publication of the above paper in the British Journal of Cancer, the authors identified an error in the formatting of their names.

(c) 2015 Cancer Research UK. All rights reserved 0007-0920/15

\section{Modification of $\mathbf{N}$ staging systems for penile cancer: a more precise prediction of prognosis}

Z-S Li, K Yao, P Chen, B Wang, J-P Chen, Q-W Mi, Y-H Li, Z-W Liu, Z-K Qin, F-J Zhou and H Han

Correction to: British Journal of Cancer (2015) 112, 1766-1771; doi:10.1038/ bjc.2015.141; published online 5 May 2015

Following the publication of this manuscript, the authors have noticed an error in Table 1.
The sixth line, which reads ' $N 2$ metastasis in multiple or bilateral inguinal lymph nodes \& Unilateral or bilateral metastasis in four or more inguinal lymph nodes without ENE, ENE of LNM or pelvic lymph nodes', should be deleted. The correct table is reproduced below.

Table 1. AJCC 7th edition $\mathbf{N}$ staging system and the modified $\mathbf{N}$ staging system

\begin{tabular}{|l|l|l}
\hline Stage & AJCC 7th edition N staging system & Modified N staging system \\
\hline
\end{tabular}

\begin{tabular}{|l|l|l|}
\hline N0 & No regional lymph node metastasis & No regional lymph node metastasis \\
\hline Nx & Regional lymph nodes cannot be assessed & Regional lymph nodes cannot be assessed \\
\hline N1 & Metastasis in a single inguinal lymph node & Metastasis in one to two unilateral inguinal lymph nodes without ENE \\
\hline N2 & $\begin{array}{l}\text { Metastasis in multiple or bilateral inguinal lymph } \\
\text { nodes }\end{array}$ & Metastasis in three unilateral or metastasis in two to three bilateral inguinal lymph nodes without ENE \\
\hline N3 & $\begin{array}{l}\text { Unilateral or bilateral ENE of LNM or pelvic lymph } \\
\text { node(s) }\end{array}$ & $\begin{array}{l}\text { Unilateral or bilateral metastasis in four or more inguinal lymph nodes without ENE, ENE of LNM or } \\
\text { pelvic lymph node(s) }\end{array}$ \\
\hline Abbreviations: ENE = extranodal extension; LNM =lymph node metastasis.
\end{tabular}

A distinct pre-existing inflammatory tumour microenvironment is associated with chemotherapy resistance in high-grade serous epithelial ovarian cancer

M Koti, A Siu, I Clément, M Bidarimath, G Turashvili, A Edwards, K Rahimi, A-M Mes-Masson and J A Squire

Correction to: British Journal of Cancer (2015) 112, 1215-1222; doi:10.1038/ bjc.2015.81; published online 12 March 2015

Upon publication of the above paper in the British Journal of Cancer, the authors noted an error in the presentation of a contributing author's surname.
The name of author 'A-M M Masson' should have been written as 'A-M Mes-Masson'. This has been corrected above.

LH McCormick Matthews, F Noble, J Tod, E Jaynes, S Harris, J N Primrose, C Ottensmeier, G J Thomas and T J Underwood

Correction to: British Journal of Cancer (2015) 113, 107-118; doi:10.1038/ bjc.2015.179; published online 25 June 2015

Following the publication of this manuscript, the authors have made arrangements to change the licence from CC-BY-NC-SA to CC-BY 4.0.

(C) 2015 Cancer Research UK. All rights reserved 0007-0920/15
Therefore the terms under which this paper is published are now altered and further details can be found on the creative commons website http:// creativecommons.org/licenses/by/4.0/ 